ZASTOSOWANIA MATEMATYKI

APPLICATIONES MATHEMATICAE

Volume jubilaire dédiè à Hugo Steinhaus

$\mathrm{X}$ (1969)

W. ŚLEB ODZINS KI (Wroclaw)

\title{
SUR DEUX GROUPES INFINIS ET CONTINUS
}

0. Ce travail consacré à deux groupes infinis et continus se compose de deux partie $\mathrm{A}$ et $\mathrm{B}$.

Dans la partie A nous appliquons la méthode initiée par $\mathrm{S}$. Lie et douée d'un fondement par E. Cartan, aux groupes des transformations conservant respectivement l'équation de Laplace et l'équation de la corde vibrante, données sur une variété analytique $V^{2}$. Dans cette étude on montre, entre autres, qu'à chacun de ces groupes est liée intrinsèquement une suite infinie et bien définie des groupes linéaires de même structure.

En se servant des résultats obtenus dans la partie $\mathbf{A}$ on construit dans la partie B deux groupes abstraits infinis et continus dont les éléments générateurs sont une variété $V^{2}$ et une suite infinie des groupes linéaires de Lie. Les considérations de cette partie présentent une application particulière de la méthode développée dans mon ouvrage [6], Ch. VI, §3.

\section{A.}

1. Préliminaires. Soit $V^{2}$ une variété connexe et orientable de classe $C^{\omega}$; supposons que sur $V^{2}$ soit donnée globalement une équation qui dans les coordonnées locales $x^{1}, x^{2}$ d'un voisinage $U \subset V^{2}$ ait la forme

$$
\frac{\partial^{2} \Theta}{\partial x^{1} \partial x^{1}}+\varepsilon \frac{\partial^{2} \Theta}{\partial x^{2} \partial x^{2}}=0, \quad \varepsilon= \pm 1
$$

On voit que pour $\varepsilon=+1$ (1.1) est l'équation de Laplace et pour $\varepsilon=-1$ l'équation de la corde vibrante.

L'équation (1.1) est invariante pour les transformations des coordonnées locales des voisinages de l'atlas de $V^{2}$ satisfaisantes aux relations

$$
\frac{\partial \bar{x}^{1}}{\partial x^{1}}=\frac{\partial \bar{x}^{2}}{\partial x^{2}}, \quad \frac{\partial \bar{x}^{1}}{\partial x^{2}}=-\varepsilon \frac{\partial \bar{x}^{2}}{\partial x^{1}} ;
$$

on voit bien que pour $\varepsilon=+1$ les relations ci-dessus forment le système des équations de Cauchy-Riemann. 
Il résulte de l'hypothèse faite sur l'équation (1.1) que les équations (1.2) sont en involution par rapport aux variables $x^{1}, x^{2}$; elles sont done les équations de définition ([1], p. 178) d'un groupe continu infini des transformations agissant sur la variété $V^{2}$; nous le désignerons par $G^{\varepsilon}$ $(\varepsilon= \pm 1)$. Posons

$$
\frac{\partial \bar{x}^{1}}{\partial x^{1}}=u_{1}^{1}, \quad \frac{\partial \bar{x}^{1}}{\partial x^{2}}=-\varepsilon u_{1}^{2}, \quad \frac{\partial \bar{x}^{2}}{\partial x^{1}}=u_{1}^{2}, \quad \frac{\partial \bar{x}^{2}}{\partial x^{2}}=u_{1}^{1},
$$

en désignant par $u_{1}^{\alpha}\left({ }^{1}\right)$ des nouvelles inconnues auxiliaires, fonctions du point $x \in U$. Les équations (1.2) peuvent être remplacées par le système (1.3) aux inconnues $\bar{x}^{x}, u_{1}^{\alpha}$.

Pour étudier le groupe $G^{\varepsilon}$ nous allons prolonger le système des équations (1.3) au moyen des différentiations. Ceci nous permettra de faire voir qu'à l'équation (1.1) sont liées intrinsèquement trois suites infinies:

1) la suite $S_{1}$ des systèmes des équations linéaires de Pfaff,

2) la suite $S_{2}$ des groupes linéaires

$$
\Gamma_{1}^{\varepsilon}, \Gamma_{2}^{\varepsilon}, \ldots, \Gamma_{r}^{e}, \ldots
$$

3) la suite $S_{3}$ des espaces fibrés principaux

$$
B_{1}^{e}, B_{2}^{e}, \ldots, B_{r}^{\varepsilon}, \ldots
$$

dont les groupes de structure sont les éléments correspondants de la suite (1.4).

2. Prologements des équations (1.3). Différentions les équations (1.3). On verra facilement que les grandeurs $\frac{\partial^{2} \bar{x}^{\kappa}}{\partial x^{\lambda} \partial x^{\mu}}$ s'expriment aux moyen des dérivées $\frac{\partial u_{1}^{\alpha}}{\partial x^{\lambda}}$, si celles-ci satisfont aux relations suivantes

$$
\frac{\partial u_{1}^{1}}{\partial x^{2}}=-\varepsilon \frac{\partial u_{1}^{2}}{\partial x^{1}}, \quad \frac{\partial u_{1}^{1}}{\partial x^{1}}=\frac{\partial u_{1}^{2}}{\partial x^{2}} .
$$

On aura alors les formules

$$
\begin{array}{rlrl}
\frac{\partial^{2} \bar{x}^{1}}{\partial x^{1} \partial x^{1}} & =\frac{\partial u_{1}^{1}}{\partial x^{1}}, & & \frac{\partial^{2} \bar{x}^{1}}{\partial x^{2} \partial x^{1}}=\frac{\partial u_{1}^{1}}{\partial x^{2}}, \\
-\frac{\partial^{2} \bar{x}^{1}}{\partial x^{1} \partial x^{2}}=-\varepsilon \frac{\partial u_{1}^{2}}{\partial x^{1}}, & \frac{\partial^{2} \bar{x}^{1}}{\partial x^{2} \partial x^{2}}=-\varepsilon \frac{\partial u_{1}^{2}}{\partial x^{2}}, \\
\frac{\partial^{2} \bar{x}^{2}}{\partial x^{1} \partial x^{1}}=\frac{\partial u_{1}^{2}}{\partial x^{1}}, & \frac{\partial^{2} \bar{x}^{2}}{\partial x^{2} \partial x^{1}}=\frac{\partial u_{1}^{2}}{\partial x^{2}}, \\
\frac{\partial^{2} \bar{x}^{2}}{\partial x^{1} \partial x^{2}}=\frac{\partial u_{1}^{1}}{\partial x^{1}}, & \frac{\partial^{2} \bar{x}^{2}}{\partial x^{2} \partial x^{2}}=\frac{\partial u_{1}^{1}}{\partial x^{2}} .
\end{array}
$$

(1) Dans tout ce travail les indices grecs $\varkappa, \lambda, \mu \ldots$ parcourent les valeurs $1,2$. 
Introduisons maintenant une seconde série des inconnues auxiliaires $u_{2}^{*}$ en posant

$$
\frac{\partial u_{1}^{1}}{\partial x^{1}}=u_{2}^{1}, \quad \frac{\partial u_{1}^{1}}{\partial x^{2}}=-\varepsilon u_{2}^{2}, \quad \frac{\partial u_{1}^{2}}{\partial x^{1}}=u_{2}^{2}, \quad \frac{\partial u_{2}^{2}}{\partial x^{2}}=u_{2}^{1}
$$

ce qui est bien compatible avec les relations (2.1). Les équations (2.2) peuvent maintenant s'écrire

$$
\begin{array}{lll}
\frac{\partial^{2} \bar{x}^{1}}{\partial x^{1} \partial x^{1}}=u_{2}^{1}, & \frac{\partial^{2} \bar{x}^{1}}{\partial x^{2} \partial x^{1}}=-\varepsilon u_{2}^{2}, \\
\frac{\partial^{2} \bar{x}^{1}}{\partial x^{1} \partial x^{2}}=-\varepsilon u_{2}^{2}, & \frac{\partial^{2} \bar{x}^{1}}{\partial x^{2} \partial x^{2}}=-\varepsilon u_{2}^{1}, \\
\frac{\partial^{2} \bar{x}^{2}}{\partial x^{1} \partial x^{1}}=u_{2}^{2}, & \frac{\partial^{2} \bar{x}^{2}}{\partial x^{2} \partial x^{1}}=u_{2}^{1}, \\
\frac{\partial^{2} \bar{x}^{2}}{\partial x^{1} \partial x^{2}}=u_{2}^{1}, & \frac{\partial^{2} \bar{x}^{2}}{\partial x^{2} \partial x^{2}}=-\varepsilon u_{2}^{2} .
\end{array}
$$

En tenant compte des formules (2.1)-(2.4) on peut se convaincre que les équations obtenues par différentiation des équations (1.3) peuvent être remplacées par les équations (2.3). Nous dirons que le dernier système des équations est le premier système prolongé des équations (1.3) qui, comme on a $v u$, remplace les équations de définition (1.2).

Nous voyons que les relations (2.3) ont la même forme que les équations (1.2); on peut donc leur appliquer le même raisonnement dont nous arons fait usage au comencement de ce $n^{0}$.

En introduisant la troisième série des inconnues auxiliaires $u_{3}^{x}$ nous obtiendrons ainsi les équations

$$
\frac{\partial u_{2}^{1}}{\partial x^{1}}=u_{3}^{1}, \quad \frac{\partial u_{2}^{1}}{\partial x^{2}}=-\varepsilon u_{3}^{1}, \quad \frac{\partial u_{2}^{2}}{\partial x^{1}}=u_{3}^{2}, \quad \frac{\partial u_{2}^{2}}{\partial x^{2}}=u_{3}^{1},
$$

qui forment le second système prolongé des équations (1.3).

En poursuivant ces raisonnements et en se servant de l'induction on obtient une suite infinie des prolongements des équations (1.3) ou, ce qui revient an même, des équations de définition (1.2) du groupe infini $G^{8}$.

Le résultat obtenu peut done être présenté sous la forme suivante

$$
\begin{aligned}
& \frac{\partial \bar{x}^{1}}{\partial x^{1}}=u_{1}^{1}, \quad \frac{\partial \bar{x}^{1}}{\partial x^{2}}=-\varepsilon u_{1}^{2}, \quad \frac{\partial \bar{x}^{2}}{\partial x^{1}}=u_{1}^{2}, \quad \frac{\partial \bar{x}^{2}}{\partial x^{2}}=u_{1}^{1}, \\
& \frac{\partial u_{r}^{1}}{\partial x^{1}}=u_{r+1}^{1}, \quad \frac{\partial u_{r}^{1}}{\partial x^{2}}=-\varepsilon u_{r+1}^{2}, \quad \frac{\partial u_{r}^{2}}{\partial x^{1}}=u_{r+1}^{2}, \quad \frac{\partial u_{r}^{2}}{\partial x^{2}}=u_{r+1}^{1}, \\
& (r=1,2,3, \ldots) \text {. }
\end{aligned}
$$


Dans ces equations $x^{1}, x^{2}$ jouent le rôle des variables indépendantes et $\bar{x}^{*}, u_{1}^{\varkappa}, u_{2}^{x}, \ldots$ sont des inconnues.

3. La suite $S_{1}$ des équations de Pfaff. Les équations (2.5) peuvent être présentées sous la forme des équations linéaires de Pfaff de la forme suivante

$$
\begin{array}{cc}
d \bar{x}^{1}=u_{1}^{1} d x^{1}-\varepsilon u_{1}^{2} d x^{2}, & d \bar{x}^{2}=u_{1}^{2} d x^{1}+u_{1}^{1} d x^{2} \\
d u_{r}^{1}=u_{r+1}^{1} d x^{1}-\varepsilon u_{r+1}^{2} d x^{2}, & d u_{r}^{2}=u_{r+1}^{2} d x^{1}+u_{r+1}^{1} d x^{2} \\
(r=1,2,3, \ldots) &
\end{array}
$$

Posons maintenant

$$
\begin{array}{cc}
\omega_{0}^{1}=u_{1}^{1} d x^{1}-\varepsilon u_{1}^{1} d x^{2}, & \omega_{0}^{2}=u_{1}^{2} d x^{1}+u_{1}^{1} d x^{2} \\
\omega_{r}^{1}=u_{r+1}^{1} d x^{1}-\varepsilon u_{r+1}^{2} d x^{2}, & \omega_{r}^{2}=u_{r+1}^{2} d x^{1}+u_{r+1}^{1} d x^{2} \\
(r=1,2,3, \ldots) .
\end{array}
$$

Il est facile de voir que le système des équations

$$
\bar{\omega}_{r}^{1}=\omega_{r}^{1}, \quad \bar{\omega}_{r}^{2}=\omega_{r}^{2} \quad(r=0,1,2, \ldots)
$$

est équivalent au système tronqué formé de $r+1$ premières des équations $\mathrm{du}$ système $(2.5)$, si l'on remplace les variables $x^{\varkappa}, u_{r}^{*}(r=1,2, \ldots)$ dans les formes $\omega$ par les variables $\bar{x}^{\varkappa}, \bar{u}_{r}^{x}$.

Il résulte de l'hypothèse que l'équation (1.1) est donnée sur toute la variété $V^{2}$ que le système des équations (1.2) est en involution par rapport aux variables $x^{1}, x^{2}$; il en est donc de même du système des équations (2.5) et de chaque système tronqué donné par les $r$ premières des équations (3.3).

4. La suite $S_{2}$ des groupes linéaires. Les formules (3.2) représentent des substitutions que l'on fait sur les différentielles $d x^{*}$ pour obtenir les formes $\omega_{r}^{\varkappa}(r=0,1,2, \ldots)$. Les matrices de ces substitutions ont la forme

$$
\gamma_{r+1}=\left[\begin{array}{rr}
u_{r+1}^{1} & -\varepsilon u_{r+1}^{2} \\
u_{r+1}^{2} & u_{r+1}^{1}
\end{array}\right] \quad(r=0,1,2, \ldots)
$$

et l'on a

$$
\text { Dét } \gamma_{r+1}=T_{r+1}=\left(u_{r+1}^{1}\right)^{2}+\varepsilon\left(u_{r+1}^{2}\right)^{2} \text {. }
$$

Si l'on y regarde les coefficients $u_{r+1}^{1}, u_{r+1}^{2}$ comme des paramètres arbitraires, les matrices $\gamma_{r_{+1}}$ forment un groupe linéaire de Lie, dont l'espace pour $\varepsilon=+1$ est l'espace euclidien à deux dimensions aux coordonnées $u_{r+1}^{1}, u_{r+1}^{2}$ et pour $\varepsilon=-1$ son domain défini par l'inégalité 
$T_{r+1}>0$. Pour la matrice inverse de (4.1) on a la formule

$$
\gamma_{r+1}^{-1}=\left[\begin{array}{rr}
\frac{u_{r+1}^{1}}{T_{r+1}} & \varepsilon \frac{u_{r+1}^{2}}{T_{r+1}} \\
-\frac{u_{r+1}^{2}}{T_{r+1}} & \frac{u_{r+1}^{1}}{T_{r+1}}
\end{array}\right] .
$$

$\mathrm{Au}$ groupe formé des matrices (4.1) nous donnerons le nom de groupe de structure d'ordre $r+1 \mathrm{du}$ groupe infini $G^{\varepsilon}$ et nous le désignerons par $\Gamma_{\boldsymbol{r}+1}^{\varepsilon}$.

Désignons respectivement par $a_{\lambda}^{x}$ et $b_{\lambda}^{x}$ les éléments des matrices (4.1) et (4.3) et calculons les formes différentielles linéaires

$$
\Pi_{\lambda}^{\varkappa}=-b_{\lambda}^{\varrho} d a_{\varrho}^{*} \text {. }
$$

Si l'on y substitue les valeurs des elements $a$ et $b$, on obtient

$$
\begin{gathered}
\Pi_{1}^{1}=-d \log \sqrt{T_{r+1}}, \quad \Pi_{1}^{2}=\frac{u_{r+1}^{2} d u_{r+1}^{1}-u_{r+1}^{1} d u_{r+1}^{2}}{T_{r+1}} \\
\Pi_{2}^{1}=\varepsilon \Pi_{1}^{2}, \quad \Pi_{2}^{2}=\Pi_{1}^{1}
\end{gathered}
$$

Posons

$$
\tau_{r+1}^{1}=-d \log \sqrt{T_{r+1}}, \quad \tau_{r_{+1}}^{2}=\frac{u_{r_{+1}}^{2} d u_{r+1}^{1}-u_{r+1}^{1} d u_{r+1}^{2}}{T_{r+1}}
$$

on aura done

$$
\Pi_{1}^{1}=\tau_{r+1}^{1}, \quad \Pi_{2}^{1}=-\varepsilon \tau_{r+1}^{2}, \quad \Pi_{1}^{2}=-\tau_{r_{+1}}^{2}, \quad \Pi_{2}^{2}=\tau_{r+1}^{1} .
$$

Il résulte des formules (4.5) que l'on a

$$
d \tau_{r+1}^{1}=0, \quad d \tau_{r_{+1}}^{2}=0 .
$$

Comme les $\tau_{r+1}^{1}, \tau_{r+1}^{2}$ sont des formes différentielles linéaires et indépendantes données dans l'espace $d u$ groupe de structure $\Gamma_{r+1}^{\varepsilon}$, ce groupe est abélien et ses constantes des structure sont toutes nulles. Il en résulte que l'algèbre du groupe $\Gamma_{r+1}^{\varepsilon}$ est aussi abélienne.

Il est facile de voir que le produit des matrices (4.1) est permutable et que par conséquence le groupe $\Gamma_{r+1}^{\varepsilon}$ est abélien. Nous avons le montré par le calcul ci-dessus pour faire apparaitre les formes $\tau$ dont nous aurons besoin dans les formules (6.8)

Nous pouvons maintenant énoncer le

ThÉoRÈme 4.1. A l'équation (1.1) donnée sur la variété $V^{2}$ est liée intrinsèquement la suite infinie des groupes linéaires

$$
\Gamma_{1}^{\varepsilon}, \Gamma_{2}^{\varepsilon}, \ldots, I_{r+1}^{\varepsilon}, \ldots
$$

qui sont des groupes abéliens de même structure. La dimension du groupe $\Gamma_{r}$ est égal à 2 et les coordonnées du point de son espace sont les nombres $u_{r}^{1}, u_{r}^{2}$. 
On a déduit la suite (4.7) des équations de la suite des équations (3.3) qui peuvent être regardées comme les prolongements de la première d'elles; parconséquent nous pouvons dire que les éléments de la suite (4.7) sont des prolongements $d u$ groupe $\Gamma_{1}^{s}$.

5. La structure de la variété $V^{2}$. D'après ce que nous avoñs supposé au $\mathrm{n}^{0} 1$, la variété $V^{2}$ est de classe $C^{\omega}$ et sur $V^{2}$ est donnée l'équation (1.1). Nous allons maintenant démontrer le

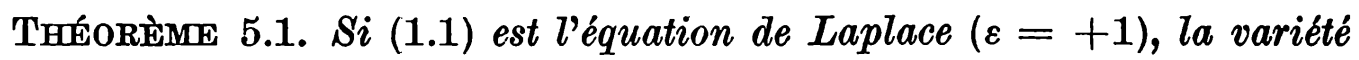
$\nabla^{2}$ a la structure complexe; si (1.1) est l'équation de la corde vibrante $(\varepsilon=-1)$, elle a la structure d'un "product space".

Considérons pour la démonstration de ce théorème un voisinage arbitraire $U$ de l'atlas de $V^{2}$ et un point $x\left(x^{*}\right) \in U$. Supposons que dans ce point soit donnée la base naturelle tangente $\left(e_{1}, e_{2}\right)$ et sa base duale $\left(f^{1}, f^{2}\right)$. Remplaçons ces bases par deux bases duales $\left(I_{1}, I_{2}\right)$ et $\left(J^{1}, J^{2}\right)$, définies au moyen des équations

$$
\boldsymbol{e}_{1}=u_{1}^{1} \boldsymbol{I}_{1}-\varepsilon u_{1}^{2} \boldsymbol{I}_{2}, \quad \boldsymbol{e}_{2}=u_{1}^{2} \boldsymbol{I}_{1}+u_{1}^{1} \boldsymbol{I}_{2}
$$

et

$$
f^{1}=\frac{u_{1}^{1}}{T_{1}} J^{1}-\frac{u_{1}^{2}}{T_{1}} J^{2}, \quad f^{2}=\frac{u_{2}^{1}}{T_{1}} J^{1}+\frac{u_{1}^{1}}{T_{1}} J^{2}
$$

dont les coefficients sont respectivement les éléments de (4.1) et de la transposée de matrice (4.2) pour $r=0$.

Les coordonnées des vecteurs $e_{1}, e_{2}$ et $f^{1}, f^{2}$ par rapport auxb ases $\left(I_{1}, I_{2}\right)$ et $\left(J^{1}, J^{2}\right)$ sont alors données au moyen des formules suivantes

$$
\begin{array}{ccc}
e_{1}^{1}=u_{1}^{1}, & e_{1}^{2}=-\varepsilon u_{1}^{2}, \quad e_{2}^{1}=u_{1}^{2}, \quad e_{2}^{2}=u_{1}^{1}, \\
f_{1}^{1}=\frac{u_{1}^{1}}{T_{1}}, \quad f_{2}^{1}=-\frac{u_{1}^{2}}{T_{1}}, \quad f_{1}^{2}=\frac{\varepsilon u_{1}^{2}}{T_{1}}, \quad f_{2}^{2}=\frac{u_{1}^{1}}{T_{1}} .
\end{array}
$$

Formons maintenant au moyen des vecteurs des bases naturelles un tenseur mixte, une fois contravariant et une fois covariant, on posant

$$
\Phi=e_{1} \otimes f^{2}-\varepsilon e_{2} \otimes f^{1},
$$

où le symbole $\otimes$ indique, comme d'habitude, le produit tensoriel.

Ses coordonnées aux bases $\left(\boldsymbol{I}_{1}, \boldsymbol{I}_{2}\right)$ et $\left(\boldsymbol{J}^{1}, \boldsymbol{J}^{2}\right)$ seront désignées par $\varphi_{x}^{\lambda}$. On aura donc d'après (5.4)

$$
\varphi_{x}^{\lambda}=e_{1}^{\lambda} f_{x}^{2}-\varepsilon e_{2}^{\lambda} f_{x}^{1} .
$$

Si l'on y introduit les valeurs données par les formules (5.3), il viendra

$$
\varphi_{1}^{1}=0, \quad \varphi_{2}^{2}=0, \quad \varphi_{2}^{1}=1, \quad \varphi_{1}^{2}=-\varepsilon .
$$

Au moyen d'un calcul facile on en déduit la relation suivante

$$
\varphi_{Q}^{\lambda} \varphi_{\kappa}^{\varrho}=-\varepsilon \delta_{\lambda}^{*}
$$


On voit done que, si $\varepsilon=+1$, le tenseur mixte $\left(\varphi_{x}^{\lambda}\right)$ est intégrable et que, par suite, la variété $V^{2}$ est analytique complexe ([3], p. 6), si $\varepsilon=-1$, elle a la structure d'un "product space".

Remarque. Dans le cas $\varepsilon=-1$ le Théorème 5.1 reste vrai sans supposer que la varieté $\nabla^{2}$ est orientable. Dans le cas $\varepsilon=+1$, ce Théorème résulte immadiatement des suppositions faites au commencement du $\mathrm{n}^{0} 1$; nous avons néanmoins fait la demonstration embrassement le deux cas pour souligner l'analogie entre les deux groupes $\mathscr{G}^{+1}$ et $\mathscr{G}^{-1}$.

6. La suite $S_{3}$. Nous allons montrer qu'on peut construire un espace fibré principal ayant la variété $V^{2}$ pour base et le groupe linéaire $\Gamma_{1}^{\varepsilon}$ $\left(\mathrm{n}^{\circ} 4\right)$ pour groupe de structure.

Imaginons pour ce but deux voisinages arbitraires $U_{i}$ et $U_{j}\left(U_{i}\right.$ $\cap U_{j} \neq 0$ ) de l'atlas de $V^{2}$ et un point arbitraire $x \epsilon U_{i} \cap U_{j}$.

Soit

$$
\gamma_{i j}=\left[\begin{array}{rr}
\alpha_{i j}^{1} & \varepsilon \alpha_{i j}^{2} \\
-\alpha_{i j}^{2} & a_{i j}^{1}
\end{array}\right]
$$

une matrice dont les éléments seraient des fonctions $C^{\omega}$ du point $x$, satisfaisant aux conditions

$$
\begin{gathered}
a_{i j}^{1}=a_{j i}^{1}, \quad a_{i j}^{2}=-a_{j i}^{2}, \quad\left(a_{i j}^{1}\right)^{2}+\varepsilon\left(a_{i j}^{2}\right)^{2}=1, \\
a_{i i}^{1}=1, \quad a_{i i}^{2}=0 .
\end{gathered}
$$

En comparant les formules (4.1) et (6.2) on voit que la matrice $\gamma_{i j}$ est un élément du groupe linéaire $\Gamma_{1}^{e}$, défini au point $x$.

En tenant compte des formules (4.1) et (4.3), où l'on doit poser $r=0$, on vérifie facilement que la matrice $\gamma_{i j}$ satisfait aux relations

$$
\gamma_{i i}=e, \quad \gamma_{i j}=\gamma_{j i}^{-1}, \quad \gamma_{j k}=\gamma_{j i} \gamma_{i k},
$$

où e désigne élément-unité du groupe $\Gamma_{1}^{\varepsilon}$ et où la dernière relation se rapporte au point $x$ commun à trois voisinages $U_{i}, V_{j}, V_{k}$ de l'atlas de $\nabla^{2}$.

On voit sur les relations (6.3) que les repères tangents à la variété $V^{2}$ forment un espace fibré principal [5] $B_{1}^{8}$ dont le groupe de structure est le groupe $\Gamma_{1}^{\varepsilon}$. Un point arbitraire de $B_{1}^{\varepsilon}$ a pour coordonnées $\left(x, u_{1}\right)\left(x \epsilon \nabla^{\mathbf{2}}\right.$, $\left.u_{1} \in \Gamma_{1}^{e}\right)$. La projection de $\left(x, u_{1}\right)$ sur la variété $\nabla^{2}$ est le point $x$ et $\Gamma_{1}^{\varepsilon}$ son groupe de structure. $V^{2}$ et $\Gamma_{1}^{s}$ étant des variétés $C^{\omega}$ il en est de même de l'espace $B_{1}^{*}$ dont la dimension est égale au produit $2 \times 2$.

Un raisonnement tout semblable à celui qui précède permet de montrer que sur la variété $B_{1}^{\varepsilon}$ on peut former un espace fibré principal $B_{2}^{\varepsilon}$ ayant $B_{1}^{\varepsilon}$ comme base et $\Gamma_{2}^{\varepsilon}$ comme groupe de structure; il faut seulement se servir des voisinages de la variété $B_{1}^{s}$ ayant pour points les systèmes $\left(x, u_{1}\right)$; la dimension de $B_{2}^{s}$ est égale à $2^{3}$. 
Au moyen de l'induction on peut ensuite former la suite des espaces fibrés principaux

$$
B_{1}^{\varepsilon}, B_{2}^{\varepsilon}, \ldots, B_{r+1}^{\varepsilon}, \ldots
$$

ayant pour groupes de structure respectivement les éléments de la suite $(4.6)$

$$
\Gamma_{1}^{\varepsilon}, \Gamma_{2}^{\varepsilon}, \Gamma_{3}^{\varepsilon}, \ldots
$$

La base de l'espace $B_{1}^{\varepsilon}$ est la variété $V^{2}$ et celle de l'espace $B_{r_{+1}}^{\varepsilon}$ est $B_{r}^{\varepsilon}$ $(r=1,2,3, \ldots)$. Le point $b_{r} \in B_{r}^{\epsilon}$ est

$$
\begin{gathered}
b_{r}=\left(x, u_{1}, u_{2}, \ldots, u_{r}\right) \\
\left(x \in V^{2}, \quad u_{1} \in \Gamma_{1}^{\varepsilon}, \quad u_{2} \in \Gamma_{2}^{\varepsilon}, \quad \ldots, \quad u_{r} \in \Gamma_{r}^{\varepsilon}\right) .
\end{gathered}
$$

Comme la variété $V^{2}$ est par hypothèse $C^{\omega}$ et les groupes (4.7) sont analytiques, il en set de même des groupes (6.4).

Dans les espaces $B_{r}^{\epsilon}$ sont donnés respectivement les systèmes des équations de Pfaff (3.3).

$$
E_{k}: \quad \bar{\omega}_{k}^{1}=\omega_{k}^{1}, \quad \bar{\omega}_{k}^{2}=\omega_{k}^{2} \quad(k=0,1,2, \ldots),
$$

où les formes $\omega_{k}^{1}, \omega_{k}^{2}$ sont définies par les formules (3.2):

$$
\begin{array}{r}
\omega_{k}^{1}=u_{k+1}^{1} d x^{1}-\varepsilon u_{k+1}^{2} d x^{2}, \quad \omega_{k}^{2}=u_{k+1}^{2} d x^{1}+u_{k+1}^{1} d x^{2} \\
(k=0,1,2, \ldots) .
\end{array}
$$

Ces systèmes sont tous en involution par rapport aux variables $x^{1}, x^{2}\left(n^{0} 3\right)$.

La solution générale $\mathrm{du}$ système $E_{0}$ a la forme $\bar{x}=\varphi(x)$ et celle $\mathrm{du}$ système $E_{0}, E_{1}, \ldots, E_{k}$ peut être écrite comme il suit

$$
\bar{x}^{k}=\varphi(x), \quad \bar{u}_{1}=\varphi\left(x, u_{1}\right), \quad \ldots, \quad \bar{u}^{k}=\varphi\left(x, u_{1}, \ldots, u_{k}\right),
$$

toutes les fonctions qui y entrent étant $C^{w}$.

Pour étudier de plus près les systèmes (6.5) différentions extérieurement les formes (3.2); on trouve

$$
\begin{gathered}
d \omega_{r}^{1}=d u_{r+1}^{1} \wedge d x^{1}-\varepsilon d u_{r+1}^{2} \wedge d x^{2} \\
d \omega_{r}^{2}=d u_{r+1}^{2} \wedge d x^{1}+d u_{r+1}^{1} \wedge d x^{2} \\
(r=0,1,2, \ldots)
\end{gathered}
$$

En résolvant les équations (6.6) par rapport aux différentielles $d x^{k}$ on obtient

$$
d x^{1}=\frac{u_{r+1}^{1}}{T_{r+1}} \omega_{r}^{1}+\varepsilon \frac{u_{r+1}^{2}}{T_{r+1}} \omega_{r}^{2}, \quad d x^{2}=-\frac{u_{r+1}^{2}}{T_{r+1}} \omega_{r}^{1}+\frac{u_{r+1}^{1}}{T_{r+1}} \omega_{r}^{2} .
$$


Si l'on porte ces expressions dans $\left(6.7^{\prime}\right)$ on trouve

$$
\begin{gathered}
d \omega_{r}^{1}=\frac{u_{r+1}^{1} d u_{r+1}^{1}+\varepsilon u_{r+1}^{2} d u_{r+1}^{2}}{T_{r+1}} \wedge \omega_{r}^{1}+\varepsilon \frac{u_{r+1}^{2} d u_{r+1}^{1}-u_{r+1}^{1} d u_{r+1}^{2}}{T_{r+1}} \wedge \omega_{r}^{2} \\
d \omega_{r}^{2}=\frac{u_{r+1}^{2} d u_{r+1}^{1}-u_{r+1}^{1} d u_{r_{+1}}^{2}}{T_{r+1}} \wedge \omega_{r}^{1}+\frac{u_{r+1}^{1} d u_{r+1}^{1}+\varepsilon u_{r+1}^{2} d u_{r+1}^{2}}{T_{r+1}} \wedge \omega_{r}^{2}
\end{gathered}
$$

En se servant des formules (4.5) on peut écrire

$$
\begin{aligned}
& d \omega_{r}^{1}=-\tau_{r+1}^{1} \wedge \omega_{r}^{1}+\varepsilon \tau_{r_{+1}}^{2} \wedge \omega_{r+1}^{2} \\
& d \omega_{r}^{2}=\tau_{r+1}^{2} \wedge \omega_{r}^{1}-\tau_{r+1}^{1} \wedge \omega_{r+1}^{2}
\end{aligned}
$$

On voit que dans ces équations caractérisant les groupes $G^{\varepsilon}$ figurent seulement des constantes $-1, \varepsilon=+1$. Elles représentent dans ces cas particuliers des nombres qui dans la théorie générale des groupes infinis de Cartan portent le nom de constantes de leur structure. Les formules (6.8) et les constantes de structure nous permettent de vérifier que tous les systèmes tronqués formés des équations (6.5) sont en involution par rapport aux variables $x^{x}$.

Les considérations de $\mathrm{n}^{\text {os }}$ précédants peuvent être résumés sous la forme suivante:

THÉORÈME 6.1. Les transformations conservant l'équation (1.1) donnée dans la variété $V^{2}$ s'obtiennent en intégrant successivement les systèmes tronqués de la suite des équations de Pfaff (6.5) qui sont toutes en involution par rapport aux variables $x^{1}, x^{2}$. Les équations de structure de ces équations sont données par (6.8).

Les formes

$$
\omega_{r}^{1}, \omega_{r}^{2} \quad(r=0,1,2, \ldots)
$$

sont définies dans les espaces fibrés principaux (6.4)

$$
B_{1}^{\varepsilon}, B_{2}^{\varepsilon}, B_{3}^{\varepsilon}, \ldots
$$

dont le premier a pour base la variété $V^{2}$ et dont chacun des autres a pour base celui qui le précède. Les groupes de structure des espaces $B_{r}^{\varepsilon}$ sont respectivement les groupes linéaires abéliens, de dimension 2, de la suite (4.7)

$$
\Gamma_{1}^{\varepsilon}, \Gamma_{2}^{\varepsilon}, \Gamma_{3}^{\varepsilon}, \ldots
$$

Le point $u_{r}$ de l'espace du groupe $\Gamma_{r}^{\varepsilon}$ a pour coordonnées $u_{r}^{1}, u_{r}^{2}$. Les formes $\omega_{r}^{1}, u_{r}^{2}$ étant linéciires et homogunes par rapport aux différentielles $d x^{1}$, $d x^{2}$ et leurs coefficients étant les coordonnées $u_{r}^{1}, u_{r}^{2}$, il en résulte que 
les solutions des systèmes tronqués de la suite (6.9) se composent de la suite des expressions

$$
\begin{array}{r}
\bar{x}=\varphi(x), \quad \bar{u}_{1}=\varphi_{1}\left(x, u_{1}\right), \quad \bar{u}_{2}=\varphi_{2}\left(x, u_{1}, u_{2}\right), \\
\quad \ldots, \\
\bar{u}_{r}=\varphi_{r}\left(x, u_{1}, \ldots, u_{r}\right) .
\end{array}
$$

B.

7. Groupes infinis de Lie-Cartan. Les résultats établis dans les $n^{\text {os }}$ précédents et résumés dans le Théorème 6.1 sont au fond un développement complété et un peu simplifié de la théorie des pseudogroupes de transformations due à E. Cartan; nous l'avons appliqué à deux cas particuliers liés aux équations (1.1). Les raisonnements dont nous avons fait usage et les notions y définies nous suggèrent la construction d'un groupe infini continu analogue a la théorie d'un groupe fini de Lie.

En se servant des notations dont nous avons fait usage dans le Théorème 6.1 posons d'abord

$$
H^{\varepsilon}=V^{2} \times \Gamma_{1}^{\varepsilon} \times \Gamma_{2}^{\varepsilon} \times \ldots,
$$

en conservant les hypothèses que nous avons faites sur les facteurs de ce produit. Il suit de ces hypothèses qu'à l'espace $H^{\varepsilon}$ peut être liée la suite (6.10) des espaces fibrés principaux et que dans ces espaces peuvent être définis les systemes $E_{k}$ des équations de Pfaff (6.5). Le point $g^{6} \in H^{\varepsilon}$ a donc une infinité des coordonnées

$$
g^{6}=\left(x, u_{1}, u_{2}, \ldots\right) \quad\left(x \in \nabla^{2}, \quad u_{1} \in \Gamma_{1}^{6}, \quad u_{2} \in \Gamma_{2}^{6}, \quad \ldots\right) .
$$

Comme un voisinage de $g^{8}$ nous allons envisager le produit cartésien des voisinages des points $x, u_{1}, u_{2}, \ldots$ dans les variétés dont ils font respectivement partie. Dans la suite, pour simplifier l'écriture, nous allons ommetre l'indice $\varepsilon$ dans le symbole (7.2).

Remarquons que la suite tronquée $x, u_{1}, u_{2}, \ldots, u_{r}$, faite des coordonnées du point (7.2), peut être regardée comme le point $b_{r}$ de l'espace fibré $B_{r}^{s}$, faisant partie de la suite (6.10), ou comme un point de l'espace $H_{r}^{\varepsilon}=V^{2} \times \Gamma_{1}^{\varepsilon} \times \Gamma_{2}^{e} \times \ldots \times \Gamma_{r}^{8}$ :

$$
g_{r}=\left(x, u_{1}, u_{2}, \ldots, u_{r}\right), \quad g_{r} \in B_{r}^{\varepsilon}, \quad g_{r} \in H_{r}^{\varepsilon} .
$$

Cette interprétation implique les formules

$$
p_{1}^{\varepsilon} g_{1}=x, \quad p_{2}^{\varepsilon} g_{2}=g_{1}, \quad \ldots, \quad p_{r}^{\varepsilon} g_{r}=g_{r-1} \quad(r=2,3, \ldots),
$$

$p_{r}^{s}$ désignant la projection propre à l'espace fibré principal $B_{r}^{s}$.

Considérons maintenant trois points ' $a$, " $a$, ," $a$ de l'espace $H^{8}$ :

$$
\begin{gathered}
' a=\left({ }^{\prime} x,{ }^{\prime} u_{1},{ }^{\prime} u_{2}, \ldots\right), \quad " \prime a=\left({ }^{\prime \prime} x,{ }^{\prime \prime} u_{1},{ }^{\prime \prime} u_{2}, \ldots\right), \\
\text { '" } a=\left({ }^{\prime \prime \prime} x,{ }^{\prime \prime \prime} u_{1},{ }^{\prime \prime \prime} u_{2}, \ldots\right)
\end{gathered}
$$


et les trois points ' $a_{r},{ }^{\prime \prime} a_{r},{ }^{\prime \prime \prime} a_{r}$ de l'espace $H_{r}^{8}$ :

$$
\begin{gathered}
{ }^{\prime} a_{r}=\left({ }^{\prime} x,{ }^{\prime} u_{1}, \ldots,{ }^{\prime} u_{r}\right), \quad "{ }^{\prime} a_{r}=\left({ }^{\prime \prime} x, " u_{1}, \ldots,{ }^{\prime \prime} u_{r}\right) \\
\text { '"' } a_{r}=\left({ }^{\prime \prime \prime} x,{ }^{\prime \prime \prime} u_{1}, \ldots,{ }^{\prime \prime \prime} u_{r}\right) .
\end{gathered}
$$

Nous dirons que "' $a$ est une fonction analytique de 'a et de "a, donnée sur $H^{8}$, si " " $a_{r}$ est une fonction analytique de ' $a_{r}$ et de " $a_{r}(r=1,2,3, \ldots)$.

Ceci posé nous pouvons dire que $H^{8}$, défini par (7.1), est une variété analytique connexe d'une infinité de dimensions; nous allons montrer que $H^{\varepsilon}$ est l'espace d'un groupe infini continu que nous désignerons par le même symbole $H^{\varepsilon}$; les points de $H^{8}$ seront appelés éléments de ce groupe.

Nous allons prouver à cet effet le suivant

THÉORÈme 7.1. Dans l'espace $H^{e}$ peut être définie sur ses points une opération, appelée multiplication, qui jouit des propriétés suivantes:

$1^{\circ} \grave{a}$ chaque paire ordonné des points $a, b \in H_{r}^{e}$ correspond un point $c$, appelé leur produit et désigné par $c=a b ; c$ est une fonction analytique de $a$ et de $b$;

$2^{\circ}$ le produit des points est associatif

$$
(a b) c=a(b c)
$$

$3^{\circ}$ il existe un point $e \epsilon B^{8}$ satisfaisant aux relations

$$
a e=e a
$$

pour tout point $a \epsilon H^{s}$; ce point porte le nom de l'unité du groupe $H^{s}$;

$4^{\circ}$ pour chaque point $a \epsilon H^{8}$ il existe un élément $a^{-1}$, appelé son inverse, satisfaisant aux conditions

$$
a a^{-1}=a^{-1} a=e ;
$$

$a^{-1}$ est une fonction analytique de a.

Pour démontrer ce théorème rappelons que tous les systèmes tronqués de la suite (6.5) sont en involution par rapport aux variables $x^{1}, x^{2}$ et que la solution générale du système $E_{0}^{s}, E_{1}^{s}, \ldots, E_{k}^{\varepsilon}$ peut être présentée sous la forme

$$
\bar{x}=\varphi(x), \quad \bar{u}_{r}=\varphi_{r}\left(x, u_{1}, \ldots, u_{r}\right) \quad(r=1,2, \ldots, k)
$$

ce qu'on peut écrire plus court

$$
\bar{g}_{k}=\Phi_{k}\left(g_{k}\right)
$$

où $g_{k}$ et $\bar{g}_{k}$ désignent des points de l'espace $B_{k}^{\varepsilon}$

$$
g_{k}=\left(x, u_{1}, \ldots, u_{k}\right), \quad \bar{g}_{k}=\left(\bar{x}, \bar{u}_{1}, \ldots, \bar{u}_{k}\right) .
$$


Considérons maintenant dans l'espace $H^{\varepsilon}$, défini par (7.1), un point fixe choisi d'ailleurs arbitrairement

$$
g^{0}=\left(x^{0}, u_{1}^{0}, u_{2}^{0}, \ldots\right)
$$

et un autre point arbitraire

$$
\bar{g}^{0}=\left(\bar{x}^{0}, \bar{u}_{1}^{0}, \bar{u}_{2}^{0}, \ldots\right) .
$$

Désignons par $\bar{g}_{k}^{0}, g_{k}^{0}$ des points de l'espace $B_{r}^{\varepsilon}$ qui ont les mêmes $k$ premières coordonnées que les points $\bar{g}^{0}, g^{0}$ :

$$
\bar{g}_{k}^{0}=\left(\bar{x}^{0}, \bar{u}_{1}^{0}, \ldots, \bar{u}_{k}^{0}\right), \quad g_{k}^{0}=\left(x^{0}, u_{1}^{0}, \ldots, u_{k}^{0}\right) .
$$

Il résulte de la théorie des systèmes des équations de Pfaff en involution qu'il existe une infinité des solutions (7.3) telles qu'il soit $\bar{g}_{k}=\bar{g}_{k}^{0}$ pour $g_{k}=g_{k}^{0}$. Soit

$$
\bar{g}_{k}=\Psi_{k}\left(g_{k}, \bar{g}_{k}^{0}\right)
$$

une telle solution; nous pouvons la choisir de manière que les équations (7.6) contiennent aussi les équations

$$
\bar{g}_{k-1}=\Psi_{k-1}\left(g_{k-1}, \bar{g}_{k-1}^{0}\right)
$$

qui représentent une solution du système $E_{0}, E_{1}, \ldots, E_{k_{-1}}$.

En appliquant ce procédé à tous les systèmes tronqués de la suite (6.5) nous obtenons une solution analytique $d u$ système infini des équations (6.5). Désignons cette solution par la formule

$$
\bar{g}=\Psi\left(g, \bar{g}^{0}\right) .
$$

Cette solution définit pour tout paire des points $g, \bar{g}^{0} \epsilon H^{\varepsilon}$ un point $\bar{g} \epsilon H^{\bullet}$. Nous dirons que la fonction $\Psi\left(g, \bar{g}^{0}\right)$ définit le produit $\bar{g}^{0} g$

$$
\bar{g}=\bar{g}^{0} g \text {. }
$$

Il résulte dela construction de (7.7) que pour $g=g^{0}$ on a $\bar{g}=\bar{g}^{0}$; donc si l'on pose $g^{0}=e$ on aura $\bar{g}^{0}=\bar{g}^{0} e$ ou $g e=g$.

Un raisonnement semblable montre qu'il est $e g=g$. Nous pouvons aussi montrer qu'à tout point $\bar{g}^{0} \epsilon H^{\varepsilon}$ correspond un autre point ' $g^{0} \epsilon H^{\varepsilon}$, tel que $\bar{g}=\Psi\left({ }^{\prime} \bar{g}^{0}, g\right)$ soit l'inverse de la transformation (7.7). Nous désignerons le point ' $\bar{g}^{0}$ par $\bar{g}_{0}^{-1}$.

Il est aussi facile de montrer que l'opération (7.8) est associative. Nous voyons ainsi que cette opération jouit de toutes les propriétés énoncées dans le Théorème 7.1 ; par conséquent l'espace $H^{\varepsilon}$ avec l'opération (7.8) définit un groupe abstrait, infini et continu.

La démonstration du Théorìme 7.1 est ainsi achevée; remarquons que le groupe $H^{\varepsilon}$ est construit au moyen de dcux élóments gónérateurs: de la vrriété $V^{2}$ et de la suite des groupes linéaires $I_{r}^{\varepsilon}(r=0,1,2, \ldots)$. 
8. Représentations du groupe abstrait $H^{\varepsilon}$. Considerons un élément $g \in H^{\varepsilon}$. La formule

$$
\bar{a}=g a \quad\left(a, \bar{a} \epsilon H^{2}\right)
$$

définit alors une transformation analytique de l'espace (7.1). Nous lui donnerons le nom d'une translation gauche et nous la désignerons par $L_{g}$. Si $g$ parcourt le groupe $H^{\varepsilon}$, les translations correspondantes $L_{g}$ forme un groupe isomorphique avec le groupe $H^{\varepsilon}$, puisque on a

$$
L_{g} L_{g^{\prime}}=L_{g g^{\prime}}, \quad L_{g^{-1}} L_{g}=L_{g} L_{g^{-1}}=L_{e}
$$

Nous désịnerons ce groupe par $H_{L}^{\varepsilon}$.

Le groupe $H_{L}^{\varepsilon}$ de translations gauches agit sur $H^{\varepsilon}$ d'une manière simplement transitive, puisque pour deux éléments arbitraires $a, \bar{a} \epsilon H^{\varepsilon}$ il existe un et un seul élément $g$ tel que $a g=\bar{a}$; il est donné par la formule $g=\bar{a}^{1} \bar{a}$. En se servant de la terminologie de S. Lie et de E. Cartan nous pouvons dire, que $H_{L}^{\varepsilon}$ est le premier groupe des paramètres de $H^{\varepsilon}$ et appeler les coordonnées de $g$ paramètres de la transformation $L_{g}$.

D'une manière analogue on peut définir, au moyen de l'égalité $\bar{a}=a g$, les translations droites dans $H^{\varepsilon}$ et leur groupe $D_{g}$.

9. Propriété du groupe infini $H^{\varepsilon}$. Soit $g=\left(x, u_{1}, u_{2}, \ldots\right)$ un point arbitraire de $H^{\varepsilon}$. Considérons une suite infinie

$$
\boldsymbol{I}=\left(\boldsymbol{I}_{0}, \boldsymbol{I}_{1}, \boldsymbol{I}_{2}, \ldots, \boldsymbol{I}_{r}, \ldots\right)
$$

des vecteurs contravariants, dont le premier est tangent à $V^{2}$ au point $x$ et dont chaque suivant $\boldsymbol{I}_{r}$ est tangent à l'espace du groupe $\Gamma_{r}^{\varepsilon}$ au point $u_{r} \in \Gamma_{r}^{\varepsilon}$. Nous allons considérer $\boldsymbol{I}$ comme un vecteur contravariant tangent à l'espace $H^{\varepsilon}$ au point $g$. Chaque suite tronquée $\left(\boldsymbol{I}_{0}, \boldsymbol{I}_{1}, \ldots, \boldsymbol{I}_{r}\right)$ peut être envisagée comme un vecteur tangent à l'espace $B_{r}^{\varepsilon}$ de la suite (6.4) au point $b_{r}=\left(x, u_{1}, u_{2}, \ldots, u_{r}\right)$.

Les vecteurs tangents à $H^{\varepsilon}$ au point $g$ forment un espace vectoriel à une infinité de dimensions. Comme la base de cet espace nous prenons un repère $R_{g}$ formé des vecteurs des espaces vectoriels tangents respectivement aux variétés $V^{2}, \Gamma_{1}^{\varepsilon}, \Gamma_{2}^{\varepsilon}, \ldots$ aux points $x, u_{1}, u_{2}, \ldots$ Si l'on adopte une orientation arbitraire pour chacun de ces espaces tangents, on établit en même temps une orientation du repère $R_{g}$. Remarquons qu'à tout repere $R_{g}$ correspond un repère dual $R_{g}^{*}$ composé des vecteurs covariants.

Considerons en particulier le repère $R_{e}$ de l'espace tang'ent au point unité $e$ de l'espace $H^{\varepsilon}$. En se servant de la translation gauche $L_{a}\left(a \in H^{\varepsilon}\right)$ on transforme le repère $R_{e}$ dans un repère $R_{a}$ au point $a$. Le point $a$ pouvant être choisi d'une manière arbitraire dans $H^{\varepsilon}$ on obtient ainsi un champs des repères orientés, invariant pour les translations gauche du groupe $H^{\varepsilon}$. Nous dirons que deux vecteurs tangents à $H^{\varepsilon}$ aux divers 
points sont équijollents, s'ils ont les mêmes coordonnées par rapport aux repères gauchement invariants. On établit ainsi un parallélisme des vecteurs dans tout l'espace $H^{\boldsymbol{b}}$. Nous pouvons donc énoncer le suivant.

THÉORÈme 9.1. Dans le groupe $H^{\varepsilon}$ il existe un parallélisme absolu.

Le groupe $H^{8}$ jouit aussi d'une autre propriété en commun avec les groupes continus finis de Lie:

THÉRÈmE 9.2. L'espace du groupe $H^{8}$ est orientable.

Ce théorème résulte $d u$ fait que tous les facteurs du produit (7.1) sont orientables.

Ajoutons enfin que par la même méthode que dans la théorie des groupes finis de Lie on peut démontrer le

THÉOR亡̀me 9.3. Le groupe infini $H^{8}$ peut être généré par un voisinage arbitraire de son unité (V. par exemple [2].)

\section{Ouvrages cités}

[1] E. Cartan, Sur la structure des groupes infinis de transformations, Ann. Ec. Norm. 21 (1904), p. $133-206$.

[2] E. Cartan, La théorie des groupes finis et continus et l'analysis situs, Mémorial des Se. Math. 42 (1950).

[3] A. Fröhlicher, Zur Differentialgeometrie der Komplexen Strukturen, Math. Ann. 129 (1955), p. 50-95.

[4] S. Lie, Gesammelte Abhandlungen, t. VI. Leipzig-Oslo 1927.

[5] N. Steenrod, The topology of fibre bundles, Princeton 1951. szawa 1963.

[6] W. Slebodziński, Formes extérieures et leurs applications, t. II, War- 